\title{
Medikamentöse Therapie von epileptischen Anfällen
} und Epilepsien

\author{
Medical treatment of acute seizures and epilepsy
}

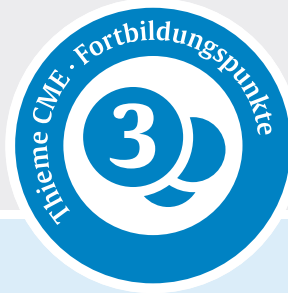

Lara Kay ${ }^{1,2}$, Sebastian Bauer ${ }^{1,2}$, Felix Rosenow ${ }^{1,2}$, Adam Strzelczyk ${ }^{1,2}$, Johann Philipp Zöllner ${ }^{1,2}$

1 Epilepsiezentrum Frankfurt Rhein-Main, Zentrum der Neurologie und Neurochirurgie, Goethe-Universität Frankfurt, Frankfurt am Main

2 LOEWE Center for Personalized Translational Epilepsy Research (CePTER), Goethe-Universität Frankfurt, Frankfurt am Main

\section{ZUSAMMENFASSUNG}

Fast jede aktive Epilepsie bedarf einer medikamentösen Therapie durch Antianfallsmedikamente (Antiiktalia). Ziel ist die Anfallsfreiheit des Patienten bei gleichzeitig guter Tolerabilität. Zur Dauertherapie fokaler Epilepsien steht eine Reihe von Antianfallsmedikamenten zur Verfügung, bei generalisierten
Epilepsien ist die Auswahl kleiner. Die Wahl des Antianfallsmedikamentes sollte neben dem Epilepsiesyndrom an den individuellen Wünschen und der Lebenssituation des Patienten, seinen Komorbiditäten und dem Nebenwirkungsprofil ausgerichtet werden. Meist wird man deshalb neuere Antianfallsmedikamente bevorzugen. Durch die Entwicklung neuer Medikamente erhöht sich die Chance einer individuell gut verträglichen und erfolgreichen Therapie. Dem gegenüber stehen höhere Therapiekosten der meisten neueren Antianfallsmedikamente. Die meisten epileptischen Anfälle sind selbstlimitierend. Ein prolongierter epileptischer Anfall muss jedoch schnell und ausreichend hoch dosiert mit Benzodiazepinen behandelt werden, um so einen therapierefraktären Verlauf abwenden zu können und das Outcome des Patienten zu verbessern.

\section{Medikamentöse Dauertherapie}

In Deutschland leiden etwa $0,6 \%$ bis $1 \%$ der Bevölkerung an Epilepsien [1-3]. Damit gehören Epilepsien zu den häufigsten neurologischen Erkrankungen in Deutschland, und auf die gesamte Lebenszeit ergibt sich ein Risiko von $2 \%$ bis $3 \%$, eine Epilepsie zu entwickeln. Die Diagnose einer Epilepsie ergibt sich durch 2 unprovozierte epileptische Anfälle oder einen epileptischen Anfall bei Vorliegen weiterer Faktoren, die das Rezidivrisiko auf $>60$ \% erhöhen. Dazu gehören zum Beispiel epilepsietypische Veränderungen im EEG oder das Vorliegen einer epileptogenen Hirnveränderung, wie nach einem Schlaganfall oder bei einem Hirntumor [4].

Aus der Diagnose einer Epilepsie ergibt sich in der Regel die Indikation zur medikamentösen Therapie. Ziel der Therapie ist dabei die Verhinderung eines Anfallsrezidives, da anhaltende Anfälle die Lebensqualität und soziale Teilhabe einschränken und zudem eine erhöhte Morbidität und Mortalität bedingen [5, 6]. Bei sehr seltenen und nicht schwerwiegenden Anfällen (z. B. <2/Jahr) oder ausschließlichen Auren ist eine Therapie eventuell verzichtbar. Etwa $60 \%$ bis $80 \%$ der Menschen mit Epilepsie werden durch eine medikamentöse Therapie anfallsfrei. Wenn es unter 2 ausreichend dosierten Antianfallsmedikamenten (synonym: Antiiktalia, auch Antiepileptika oder Antikonvulsiva genannt) nicht zu einer Anfallsfreiheit kommt, liegt eine pharmakoresistente Epilepsie vor [7, 8]. Dann sollte eine epilepsiechirurgische Therapieoption geprüft werden [9]. Alle gängigen Medikamente unterdrücken ausschließlich das Auftreten epileptischer Anfälle und wirken somit rein symptomatisch. Im Englischen wird deshalb neuerdings der Begriff „anti-seizure drug“ verwendet (Äquivalent zu Antianfallsmedikament), der oft gebrauchte Begriff Antiepileptika ist somit irreführend. Die Auswahl eines Antianfallsmedikamentes erfolgt nach dem Epilepsiesyndrom (fokal oder generalisiert), dem Alter des Patienten und bekannten Komorbiditäten. Zudem sind die angenommene Wirksamkeit und mögliche Nebenwirkungen zu bedenken.

Die Zahl der verfügbaren Antianfallsmedikamente hat sich, mit zunehmender Dynamik seit den 1980er-Jahren, stetig erhöht ( $\bullet$ Abb. 1). Eine Wirküberlegenheit einzelner Medikamente konnte dabei nur in Ausnahmefällen nachgewiesen werden. In der Regel zeigte sich keine bessere Wirksamkeit als bei Antianfallsmedikamenten der ersten und zweiten Generation ( $\triangleright$ Abb. 1). Eine Metaanalyse fand keine Wirksamkeitsunterschiede der neueren Medikamente Brivaracetam, Perampanel und Lacosamid [10]. Ältere Metaanalysen favorisierten teils Topiramat [11,12] oder Levetiracetam [13]. Üblicherweise werden neue Antianfallsmedikamente zunächst zur Zusatzbehandlung von Patienten mit fokalen Epilepsien zugelassen [14]. Primärer Endpunkt ist dabei die Reduktion der Anfallsfrequenz um $50 \%$ (,,50\%-Responderrate“). Eine Zulassung zur initialen Monotherapie erfolgt durch die EMA erst nach Studien mit Darstellung einer Nichtunterlegenheit (engl.: Non-inferiority) gegenüber Carbamazepin oder einem anderen Standardmedikament bei fokalen Epilepsien. 


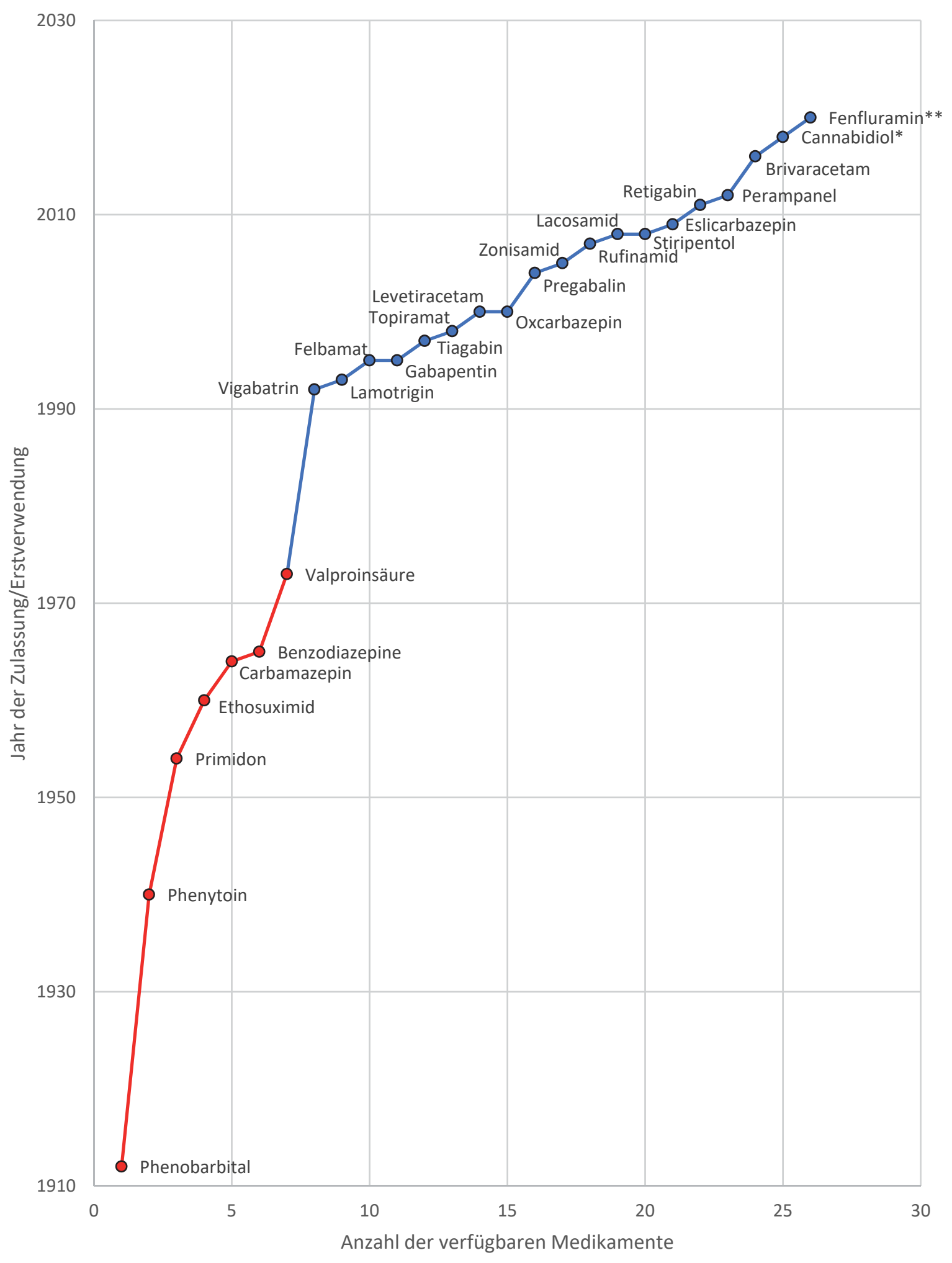

Abb. 1 Antianfallsmedikamente bis zum Jahr 2020, Daten aus [14]; Antianfallsmedikamente der ersten und zweiten Generation rot, solche der dritten Generation blau markiert. * Cannabidiol 2019 durch die amerikanische Food and Drug Administration zugelassen. ${ }^{* *}$ Zulassung von Fenfluramin [80] für 2020 erwartet. 
In solchen Studien zur initialen Monotherapie oder der offenen SANAD-Studie mit direktem Vergleich von Medikamenten zeigte sich bei fokalen Epilepsien eine bessere Wirksamkeit von Carbamazepin gegenüber Gabapentin [15]. Levetiracetam [16] und Zonisamid [17] waren Carbamazepin nicht unterlegen. Lamotrigin war Carbamazepin in der Wirksamkeit nicht unterlegen, aber besser verträglich [15]. Bei generalisierten und unklassifizierten Epilepsien war Valproat besser verträglich als Topiramat und wirksamer als Lamotrigin [18]. Bei der kindlichen Absencenepilepsie waren Ethosuximid und Valproat wirksamer als Lamotrigin, Ethosuximid war dabei verträglicher als Valproat [19].

Einige neuere Antianfallsmedikamente sind besser verträglich als solche der ersten und zweiten Generation. Bei Überdosierungen können jedoch ebenfalls Schwindel, Doppelbilder oder Übelkeit auftreten. Ein Vorteil kann sein, dass einige neuere Medikamente wie Lacosamid, Levetiracetam, Gabapentin und Pregabalin nicht und andere, wie Brivaracetam oder Zonisamid, nur wenig in klinisch relevantem Ausmaß mit anderen Medikamenten pharmakokinetisch interagieren. Ein therapeutisches Drug Monitoring ist bei den meisten neueren Antianfallsmedikamenten im Regelfall nicht nötig. Ausnahmen stellen die Lamotrigineinnahme in der Schwangerschaft sowie der Verdacht auf Noncompliance, Intoxikation, Interaktion oder bekannte Eliminationseinschränkungen z. B. bei Leber- oder Niereninsuffizienz dar.

\section{Gebräuchliche Substanzen}

Im Folgenden werden die gebräuchlichen Substanzen vorgestellt, die Anordnung richtet sich nach den Wirkmechanismen ( $\triangleright$ Tab. 1).

Carbamazepin (CBZ) war das erste Antianfallsmedikament der Dibenzazepin-Gruppe (CBZ, OXC, ESL) und verlängert den inaktivierten Zustand spannungsabhängiger Natriumkanäle. Es ist zur Therapie fokaler Epilepsien bei Kindern und Erwachsenen zugelassen und war hierfür lange die Leitsubstanz. Für CBZ liegt somit eine lange klinische Erfahrung vor. Als CYP-Induktor führt es zu sehr breiten Arzneimittelinteraktionen, u. a. schränkt es die Wirksamkeit hormoneller Kontrazeptiva ein. Die erste Zieldosis bei Erwachsenen beträgt $400 \mathrm{mg} / \mathrm{d}$, die zugelassene Höchstdosis liegt bei $1200 \mathrm{mg} / \mathrm{d}$. Die Retard-Formulierung sollte bevorzugt werden. Die Ein-Jahres-Anfallsfreiheit bei neudiagnostizierter Epilepsie beträgt etwa 73\% [16]. Bei Personen asiatischer Abstammung mit HLA-B*1502-Allel (z. B. $10 \%$ der Han-Chinesen, Thailänder) ist das Risiko schwerer allergischer Hautreaktionen stark erhöht, deshalb sollte bei diesen Patienten vor Therapiebeginn eine HLA-Bestimmung erfolgen. Auch das HLA-A*3101-Allel bei manchen Europäern und Japanern prädisponiert für allergische Hautreaktionen, eine allgemeine HLA-Testung wird für diese Gruppen aber derzeit nicht empfohlen (siehe auch Artikel von S. Wolking und H. Lerche in dieser Ausgabe). Weitere Nebenwirkungen können Herzrhythmusstörungen und Blutbildveränderungen sein.

Oxcarbazepin (OXC) ist zur Mono- und Kombinationstherapie fokaler Epilepsien ab dem 6. Lebensjahr zugelassen. Es wirkt, ähnlich wie Carbamazepin, vor allem als Natriumkanalblocker, jedoch auf die langsame Inaktivierung. OXC wird in der Leber zum aktiven S-10-Monohydroxy- Metaboliten (MHD) reduziert, das chemisch dem S-Licarbazepin entspricht und eine Halbwertszeit von 9 Stunden besitzt. OXC erhöht die Halbwertszeit von PHB und PHT und verringert die Halbwertszeit von CBZ sowie den Serumspiegel oraler Kontrazeptiva. Die maximale zugelassene Tagesdosis bei Erwachsenen liegt bei $2400 \mathrm{mg} / \mathrm{d}$ in 2 Einzelgaben bei Retardpräparaten, begonnen wird üblicherweise mit 300-600 mg/d. Die Wirkstärke in der Add-on-Therapie war nicht unterschiedlich zu CBZ, VPA und PHT (Responderrate ca. $40 \%$ ). $57 \%$ der Menschen mit einer neudiagnostizierten Epilepsie waren nach 12 Monaten OXC-Monotherapie anfallsfrei [20, 21]. In Ergebnissen des EURAP-Schwangerschaftsregisters führte die OXC-Monotherapie nicht zu einem erhöhten Risiko schwerer Kindsfehlbildungen, bei einer allerdings zu niedrigen Fallzahl für eine abschließende Beurteilung [22]. Eine spezifische UAW von OXC stellt die Hyponatriämie dar. OXC ist eine wirksame Therapiealternative bei fokalen Epilepsien. Insbesondere bei älteren Patienten sollte auf das Hyponatriämierisiko geachtet werden [23]. Es sollte primär die retardierte Formulierung von OXC eingesetzt werden.

Eslicarbazepin-Acetat (ESL) ist zugelassen zur Monotherapie fokaler Epilepsien bei Erwachsenen, als Zusatztherapie ab dem 6. Lebensjahr. ESL ist ein Prodrug und wird in der Leber vorwiegend zum S-Enantiomer des Licarbazepin (S-Licarbazepin, entspricht dem wirksamen Metaboliten von OXC) metabolisiert. Der Wirkmechanismus entspricht deshalb dem von OXC. Zudem wurde eine Wirkung auf T-Typ-Kalziumkanäle gezeigt [24]. Die Halbwertszeit von ESL beträgt 20-24 Stunden. ESL erniedrigt die Plasmakonzentration von oralen Kontrazeptiva und von Simvastatin. PB, PHT und CBZ erhöhen die Clearance von ESL. Die Add-On-Responderrate lag bei $53 \%$. Die zugelassene Zieldosis beträgt 800-1200 mg und kann einmal täglich abends eingenommen werden. Bei Monotherapie können bis 1600 mg verwendet werden. Die erste Dosis beträgt üblicherweise $400 \mathrm{mg}$. ESL war in der Monotherapie CBZ-Retard nicht unterlegen. Ein Vorteil von ESL gegenüber CBZ und OXC ist die einmal tägliche Einnahme, eine Wirküberlegenheit ist nicht bewiesen [25]. Eine spezifische UAW von ESL stellt - wie bei OXC - die Hyponatriämie dar, insbesondere bei älteren Patienten sollte auf das Hyponatriämierisiko geachtet werden [23].

Lacosamid (LCM) wirkt wie OXC und ESL antikonvulsiv über eine Verstärkung der langsamen Inaktivierung spannungsabhängiger Natriumkanäle. LCM ist zugelassen für 
• Tab. 1 Übersicht über zur Dauertherapie verwendeter Antianfallsmedikamente.

\begin{tabular}{|c|c|c|c|c|c|c|c|c|c|}
\hline Name & Code & $\begin{array}{l}\text { Mechanis- } \\
\text { mus }\end{array}$ & Indikation & $\begin{array}{l}\text { Startdosis } \\
\text { (Erwach- } \\
\text { sene) }\end{array}$ & $\begin{array}{l}\text { Steigerung } \\
\text { (Erwach- } \\
\text { sene) }\end{array}$ & $\begin{array}{l}\text { Zieldosis } \\
\text { bis max. } \\
\text { zugelassene } \\
\text { Tagesdosis } \\
\text { (Erwachsene) }\end{array}$ & $\begin{array}{l}\text { Wichtigste } \\
\text { spezifische UAW }\end{array}$ & $\begin{array}{l}\text { Interak- } \\
\text { tions- } \\
\text { poten- } \\
\text { zial }\end{array}$ & $\begin{array}{l}\text { UAW/ } \\
\text { Bemerkungen }\end{array}$ \\
\hline $\begin{array}{l}\text { Carba- } \\
\text { mazepin }\end{array}$ & CBZ & $\begin{array}{l}\mathrm{Na+-Kanal-} \\
\text { blockade }\end{array}$ & $\begin{array}{l}\text { Monothera- } \\
\text { pie fokaler } \\
\text { Anfälle }\end{array}$ & $\begin{array}{l}200- \\
300 \mathrm{mg} / \mathrm{d}\end{array}$ & $\begin{array}{l}\text { z. B. wö- } \\
\text { chentlich } \\
\text { um } 200 \text { bis } \\
300 \mathrm{mg} / \mathrm{d}\end{array}$ & $400-1200 \mathrm{mg} / \mathrm{d}$ & $\begin{array}{l}\text { Hyponatriämie, } \\
\text { Bradykardie/AV- } \\
\text { Block, Leuko- } \\
\text { penie (häufig), } \\
\text { Agranulozytose } \\
\text { (sehr selten), Ste- } \\
\text { vens-Johnson-Syn- } \\
\text { drom, Osteoporo- } \\
\text { se/Osteopenie }\end{array}$ & ++ & $\begin{array}{l}\text { Erhöhtes Risiko } \\
\text { für Steven-John- } \\
\text { son-Syndrom bei } \\
\text { HLA-B*1502 (v. a. } \\
\text { Han-Chinesen, } \\
\text { Thailänder): ge- } \\
\text { netische Testung } \\
\text { vor Therapiebe- } \\
\text { ginn notwendig; } \\
\text { Risiko auch bei } \\
\text { HLA-A*3101 (v. a. } \\
\text { Japaner, Europäer) }\end{array}$ \\
\hline $\begin{array}{l}\text { Oxcar- } \\
\text { bazepin }\end{array}$ & OXC & $\begin{array}{l}\mathrm{Na+-Kanal-} \\
\text { blockade }\end{array}$ & $\begin{array}{l}\text { Monothera- } \\
\text { pie fokaler } \\
\text { Anfälle }\end{array}$ & $\begin{array}{l}300- \\
600 \mathrm{mg} / \mathrm{d}\end{array}$ & $\begin{array}{l}\text { z. B. wö- } \\
\text { chentlich } \\
\text { um } 450- \\
600 \mathrm{mg} / \mathrm{d}\end{array}$ & $600-2400 \mathrm{mg} / \mathrm{d}$ & $\begin{array}{l}\text { Hyponatriämie, } \\
\text { AV-Block }\end{array}$ & + & $\begin{array}{l}\text { Kreuzreaktion } \\
\text { hinsichtlich Aller- } \\
\text { gie bei } 25-30 \% \text {; } \\
\text { ebenfalls geneti- } \\
\text { sche Testung von } \\
\text { Patienten asiati- } \\
\text { scher Herkunft } \\
\text { auf HLA-B*1502 } \\
\text { empfohlen }\end{array}$ \\
\hline $\begin{array}{l}\text { Eslicar- } \\
\text { bazepin }\end{array}$ & ESL & $\begin{array}{l}\mathrm{Na+-Kanal-} \\
\text { blockade }\end{array}$ & $\begin{array}{l}\text { Fokale } \\
\text { Anfälle }\end{array}$ & $400 \mathrm{mg} / \mathrm{d}$ & $\begin{array}{l}\text { z. B. alle } \\
1-2 \text { Wo- } \\
\text { chen um } \\
400 \mathrm{mg} / \mathrm{d}\end{array}$ & $400-1600 \mathrm{mg} / \mathrm{d}$ & $\begin{array}{l}\text { Hyponatriämie, } \\
\text { AV-Block }\end{array}$ & + & $\begin{array}{l}\text { ebenfalls geneti- } \\
\text { sche Testung von } \\
\text { Patienten asiati- } \\
\text { scher Herkunft } \\
\text { auf HLA-B*1502 } \\
\text { empfohlen, analog } \\
\text { zu CBZ und OXC }\end{array}$ \\
\hline $\begin{array}{l}\text { Lacosa- } \\
\text { mid }\end{array}$ & $\begin{array}{l}\text { LCM, } \\
\text { LCS }\end{array}$ & $\begin{array}{l}\mathrm{Na}+- \text { Kanal- } \\
\text { blockade } \\
\text { (slow inacti- } \\
\text { vation) }\end{array}$ & $\begin{array}{l}\text { Fokale An- } \\
\text { fälle, auch } \\
\text { Monothe- } \\
\text { rapie }\end{array}$ & $100 \mathrm{mg} / \mathrm{d}$ & $\begin{array}{l}\text { z. B. } \\
\text { wöchent- } \\
\text { lich um } \\
100 \mathrm{mg} / \mathrm{d}\end{array}$ & $\begin{array}{l}200-600 \mathrm{mg} / \mathrm{d} \\
\text { (auch i.v. vorh.) }\end{array}$ & AV-Block & - & $\begin{array}{l}\text { EKG-Kontrolle v. a. } \\
\text { bei bekannten } \\
\text { Herzrhythmus- } \\
\text { störungen }\end{array}$ \\
\hline $\begin{array}{l}\text { Lamotri- } \\
\text { gin }\end{array}$ & LTG & $\begin{array}{l}\mathrm{Na+-Kanal-} \\
\text { blockade }\end{array}$ & $\begin{array}{l}\text { Monothera- } \\
\text { pie fokaler } \\
\text { und gene- } \\
\text { ralisierter } \\
\text { Anfälle }\end{array}$ & $\begin{array}{l}25 \mathrm{mg} / \mathrm{d} \\
(12,5 \mathrm{mg} / \mathrm{d} \\
\text { bei } \\
\text { VPA-Thera- } \\
\text { pie) }\end{array}$ & $\begin{array}{l}25 \mathrm{mg} / \mathrm{d} \text { alle } \\
2 \text { Wochen } \\
\text { (langsamer } \\
\text { bei VPA-Ein- } \\
\text { nahme) }\end{array}$ & $\begin{array}{l}100-400 \mathrm{mg} / \mathrm{d} \\
\text { (im Einzelfall } \\
\text { und nach } \\
\text { Verträglichkeit } \\
\text { bis } 600 \mathrm{mg} / \mathrm{d} \\
\text { möglich) }\end{array}$ & $\begin{array}{l}\text { Allergische } \\
\text { Hautreaktion } \\
\text { (Stevens-Johnson- } \\
\text { Syndrom } 0,1 \% \text { ) }\end{array}$ & \pm & $\begin{array}{l}\text { Langsame Aufdo- } \\
\text { sierung ( } 25 \mathrm{mg} / \mathrm{d} \\
\text { alle } 2 \text { Wochen) } \\
\text { zum Erkennen und } \\
\text { zur Risikoreduk- } \\
\text { tion allergischer } \\
\text { Reaktionen; } \\
\text { Halbierung der } \\
\text { Geschwindig- } \\
\text { keit, Start- und } \\
\text { Zieldosis bei } \\
\text { VPA-Therapie; } \\
\text { Interaktion mit } \\
\text { oralen Kontrazep- } \\
\text { tiva (wechselseiti- } \\
\text { ge Wirkabschwä- } \\
\text { chung) }\end{array}$ \\
\hline
\end{tabular}


- Tab. 1 Fortsetzung.

\begin{tabular}{|c|c|c|c|c|c|c|c|c|c|}
\hline Name & Code & $\begin{array}{l}\text { Mechanis- } \\
\text { mus }\end{array}$ & Indikation & $\begin{array}{l}\text { Startdosis } \\
\text { (Erwach- } \\
\text { sene) }\end{array}$ & $\begin{array}{l}\text { Steigerung } \\
\text { (Erwach- } \\
\text { sene) }\end{array}$ & $\begin{array}{l}\text { Zieldosis } \\
\text { bis max. } \\
\text { zugelassene } \\
\text { Tagesdosis } \\
\text { (Erwachsene) }\end{array}$ & $\begin{array}{l}\text { Wichtigste } \\
\text { spezifische UAW }\end{array}$ & $\begin{array}{l}\text { Interak- } \\
\text { tions- } \\
\text { poten- } \\
\text { zial }\end{array}$ & $\begin{array}{l}\text { UAW/ } \\
\text { Bemerkungen }\end{array}$ \\
\hline $\begin{array}{l}\text { Pheny- } \\
\text { toin }\end{array}$ & PHT & $\begin{array}{l}\mathrm{Na}+- \text { Kanal- } \\
\text { blockade }\end{array}$ & $\begin{array}{l}\text { Monothera- } \\
\text { pie fokaler } \\
\text { Anfälle }\end{array}$ & $100 \mathrm{mg} / \mathrm{d}$ & $\begin{array}{l}\text { z. B. wö- } \\
\text { chentlich } \\
\text { um } 50 \mathrm{md} / \mathrm{d} \\
\text { (Spiegel- } \\
\text { kontrolle!) }\end{array}$ & $\begin{array}{l}200-400 \mathrm{mg} \\
\text { (auch i.v. vorh.) }\end{array}$ & $\begin{array}{l}\text { Irreversible } \\
\text { Kleinhirnatrophie, } \\
\text { Polyneuropathie, } \\
\text { Gingivahyper- } \\
\text { plasie, Herzrhyth- } \\
\text { musstörungen, } \\
\text { AV-Block, Hirsu- } \\
\text { tismus, Osteopo- } \\
\text { rose, Bradykardie } \\
\text { und Hypotension } \\
\text { bei i.v.-Gabe }\end{array}$ & ++ & $\begin{array}{l}\text { Nicht mehr erste } \\
\text { Wahl wegen häu- } \\
\text { figer Nebenwir- } \\
\text { kungen, hohem } \\
\text { Interaktions- } \\
\text { potenzial, nicht } \\
\text { linearer Kinetik; } \\
\text { Spiegelkontrollen } \\
\text { bei Dosisänderung } \\
\text { sinnvoll; Vorsicht } \\
\text { bei i.v.-Gabe } \\
\text { über periphere } \\
\text { Venenzugänge } \\
\text { (Gewebsnekrosen } \\
\text { bei Paravasat) }\end{array}$ \\
\hline $\begin{array}{l}\text { Levetira- } \\
\text { cetam }\end{array}$ & LEV & $\begin{array}{l}\text { Einfluss auf } \\
\text { synaptische } \\
\text { Funktion } \\
\text { durch } \\
\text { Bindung } \\
\text { an präsyn. } \\
\text { SV2A-Protein }\end{array}$ & $\begin{array}{l}\text { Monothera- } \\
\text { pie fokaler } \\
\text { Anfälle, ge- } \\
\text { neralisierte } \\
\text { Anfälle } \\
\text { (JME, IGE } \\
\text { mit GTKA) }\end{array}$ & $\begin{array}{l}500- \\
1000 \mathrm{mg} / \mathrm{d}\end{array}$ & $\begin{array}{l}\text { z. B. alle } \\
3-7 \text { Tage } \\
\text { um } \\
500 \mathrm{mg} / \mathrm{d}\end{array}$ & $\begin{array}{l}1000- \\
3000 \mathrm{mg} / \mathrm{d} \\
\text { (auch i.v. vorh.) }\end{array}$ & $\begin{array}{l}\text { Aggressivität, } \\
\text { Depression, Atem- } \\
\text { wegsinfekte }\end{array}$ & - & $\begin{array}{l}\text { Häufig eingesetzt, } \\
\text { breit wirksam, } \\
\text { kann schnell auf- } \\
\text { dosiert werden, } \\
\text { Zurückhaltung bei } \\
\text { psychiatrischen } \\
\text { Begleiterkrankun- } \\
\text { gen, insbesondere } \\
\text { Depression }\end{array}$ \\
\hline $\begin{array}{l}\text { Brivara- } \\
\text { cetam }\end{array}$ & BRV & $\begin{array}{l}\text { Einfluss auf } \\
\text { synaptische } \\
\text { Funktion } \\
\text { durch } \\
\text { Bindung } \\
\text { an präsyn. } \\
\text { SV2A-Protein }\end{array}$ & $\begin{array}{l}\text { Fokale } \\
\text { Anfälle }\end{array}$ & $50 \mathrm{mg} / \mathrm{d}$ & $\begin{array}{l}\text { z. B. alle } \\
\text { 3-7 Tage } \\
\text { um } 25 \mathrm{mg} / \mathrm{d}\end{array}$ & $\begin{array}{l}50-200 \mathrm{mg} / \mathrm{d} \\
\text { (auch i.v. vorh.) }\end{array}$ & $\begin{array}{l}\text { Aggressivität, } \\
\text { Depression, Atem- } \\
\text { wegsinfekte }\end{array}$ & \pm & - \\
\hline Valproat & VPA & $\begin{array}{l}\mathrm{Na}+- \text { und } \\
\mathrm{Ca}^{2+}-\text { Kanal- } \\
\text { blockade, } \\
\text { Hemmung } \\
\text { des GABA- } \\
\text { Abbaus }\end{array}$ & $\begin{array}{l}\text { Monothera- } \\
\text { pie fokaler } \\
\text { und gene- } \\
\text { ralisierter } \\
\text { Anfälle }\end{array}$ & $\begin{array}{l}300- \\
500 \mathrm{mg} / \mathrm{d} \\
\text { (nach KG) }\end{array}$ & $\begin{array}{l}\text { z. B. } \\
300 \mathrm{mg} \text { alle } \\
4-7 \text { Tage } \\
\text { (nach KG, } \\
\text { Spiegelkon- } \\
\text { trolle!) }\end{array}$ & $\begin{array}{l}600-1500 \mathrm{mg} \\
(-2000 \mathrm{mg}) \\
\text { (auch i.v. vorh.) }\end{array}$ & $\begin{array}{l}\text { Tremor, Ge- } \\
\text { wichtszunahme, } \\
\text { polyzystische Ova- } \\
\text { rien, Haarausfall, } \\
\text { Blutgerinnungs- } \\
\text { störung, Leber- } \\
\text { schädigung, Hy- } \\
\text { perammonämie, } \\
\text { Parkinson-Syn- } \\
\text { drom, Pankreati- } \\
\text { tis, Osteoporose }\end{array}$ & ++ & $\begin{array}{l}\text { Breit wirksam, } \\
\text { hohes Interak- } \\
\text { tionspotenzial, } \\
\text { hohes Teratogeni- } \\
\text { tätsrisiko, deshalb } \\
\text { bei gebärfähigen } \\
\text { Frauen nicht } \\
\text { einsetzen }\end{array}$ \\
\hline $\begin{array}{l}\text { Zonisa- } \\
\text { mid }\end{array}$ & ZNS & $\begin{array}{l}\mathrm{Na}^{+}-\text {und } \\
\mathrm{Ca}^{2+}-\text { Kanal- } \\
\text { blockade, } \\
\text { Carboanhy- } \\
\text { drasehem- } \\
\text { mung }\end{array}$ & $\begin{array}{l}\text { Monothera- } \\
\text { pie fokaler } \\
\text { Anfälle }\end{array}$ & $50 \mathrm{mg} / \mathrm{d}$ & $\begin{array}{l}\text { alle } 2 \text { Wo- } \\
\text { chen um } \\
100 \mathrm{mg} / \mathrm{d} \text { in } \\
\text { der Mono- } \\
\text { therapie }\end{array}$ & $200-500 \mathrm{mg} / \mathrm{d}$ & $\begin{array}{l}\text { Gewichtsverlust, } \\
\text { Konzentrati- } \\
\text { onsstörung, } \\
\text { andere psychiat- } \\
\text { rische Symptome, } \\
\text { Nierensteine, } \\
\text { vermindertes } \\
\text { Schwitzen, Glau- } \\
\text { kom, Parästhesi- } \\
\text { en, metabolische } \\
\text { Azidose }\end{array}$ & \pm & $\begin{array}{l}\text { Hyperthermie bei } \\
\text { Anhidrose möglich }\end{array}$ \\
\hline
\end{tabular}


> Tab. 1 Fortsetzung.

\begin{tabular}{|c|c|c|c|c|c|c|c|c|c|}
\hline Name & Code & $\begin{array}{l}\text { Mechanis- } \\
\text { mus }\end{array}$ & Indikation & $\begin{array}{l}\text { Startdosis } \\
\text { (Erwach- } \\
\text { sene) }\end{array}$ & $\begin{array}{l}\text { Steigerung } \\
\text { (Erwach- } \\
\text { sene) }\end{array}$ & $\begin{array}{l}\text { Zieldosis } \\
\text { bis max. } \\
\text { zugelassene } \\
\text { Tagesdosis } \\
\text { (Erwachsene) }\end{array}$ & $\begin{array}{l}\text { Wichtigste } \\
\text { spezifische UAW }\end{array}$ & $\begin{array}{l}\text { Interak- } \\
\text { tions- } \\
\text { poten- } \\
\text { zial }\end{array}$ & $\begin{array}{l}\text { UAW/ } \\
\text { Bemerkungen }\end{array}$ \\
\hline $\begin{array}{l}\text { Topira- } \\
\text { mat }\end{array}$ & TPM & $\begin{array}{l}\text { AMPA-Rezep- } \\
\text { torblockade, } \\
\text { GABAerg, } \\
\text { Carbo- } \\
\text { anhydrase- } \\
\text { hemmung, } \\
\text { Na+-Kanal- } \\
\text { blockade }\end{array}$ & $\begin{array}{l}\text { Monothera- } \\
\text { pie fokaler } \\
\text { und gene- } \\
\text { ralisierter } \\
\text { Anfälle }\end{array}$ & $25 \mathrm{mg} / \mathrm{d}$ & $\begin{array}{l}\text { z. B. wö- } \\
\text { chentlich } \\
\text { um } 25 \mathrm{mg} / \mathrm{d}\end{array}$ & $50-500 \mathrm{mg} / \mathrm{d}$ & $\begin{array}{l}\text { Gewichtsverlust, } \\
\text { Konzentrations- } \\
\text { und Wortfin- } \\
\text { dungsstörungen, } \\
\text { andere psychiat- } \\
\text { rische Symptome, } \\
\text { Nierensteine, ver- } \\
\text { mindertes Schwit- } \\
\text { zen, Glaukom, } \\
\text { Parästhesien, } \\
\text { metabolische Azi- } \\
\text { dose, Pankreatitis, } \\
\text { Haarausfall }\end{array}$ & \pm & $\begin{array}{l}\text { Zusätzliche Wir- } \\
\text { kung zur Migräne- } \\
\text { prophylaxe }\end{array}$ \\
\hline Sultiam & STM & $\begin{array}{l}\text { Carbo- } \\
\text { anhydrase- } \\
\text { hemmung, } \\
\text { Na+-Kanal- } \\
\text { blockade }\end{array}$ & $\begin{array}{l}\text { Nur } \\
\text { Rolando- } \\
\text { Epilepsie } \\
\text { (BCECTS) }\end{array}$ & $\begin{array}{l}\text { Nach KG } \\
(5-10 \mathrm{mg} / \\
\text { kg KG/d) }\end{array}$ & & $200-400 \mathrm{mg} / \mathrm{d}$ & $\begin{array}{l}\text { Gewichtsverlust, } \\
\text { Verhaltensstö- } \\
\text { rung, Halluzinatio- } \\
\text { nen, Parästhesien, } \\
\text { Tachypnoe }\end{array}$ & + & - \\
\hline $\begin{array}{l}\text { Gaba- } \\
\text { pentin }\end{array}$ & GBP & $\begin{array}{l}\text { Bindung an } \\
\mathrm{Ca}^{2+}-\mathrm{Kanal}- \\
\text { protein }\end{array}$ & $\begin{array}{l}\text { Monothe- } \\
\text { rapie fokale } \\
\text { Anfälle }\end{array}$ & $300 \mathrm{mg} / \mathrm{d}$ & $\begin{array}{l}300 \mathrm{mg} / \mathrm{d} \text { in } \\
\text { den ersten } \\
3 \text { Tagen, } \\
\text { dann } \\
\text { z. B. um } \\
300 \mathrm{mg} / \mathrm{d} \\
\text { alle } \\
\text { 2-3 Tage }\end{array}$ & $900-3600 \mathrm{mg} / \mathrm{d}$ & $\begin{array}{l}\text { Psychiatrische } \\
\text { Störungen, Ge- } \\
\text { wichtszu- oder - } \\
\text { abnahme, Ödeme, } \\
\text { selten Pankreatitis }\end{array}$ & - & $\begin{array}{l}\text { Wahrscheinlich } \\
\text { nur geringe } \\
\text { antikonvulsive } \\
\text { Effektivität }\end{array}$ \\
\hline $\begin{array}{l}\text { Prega- } \\
\text { balin }\end{array}$ & PGB & $\begin{array}{l}\text { Bindung an } \\
\mathrm{Ca}^{2+}-\mathrm{Kanal}- \\
\text { protein }\end{array}$ & $\begin{array}{l}\text { Fokale } \\
\text { Anfälle }\end{array}$ & $150 \mathrm{mg} / \mathrm{d}$ & $\begin{array}{l}\text { z. B. um } \\
150 \mathrm{mg} / \mathrm{d} \\
\text { nach der } \\
1 . \text { Wo- } \\
\text { che, um } \\
300 \mathrm{mg} / \mathrm{d} \\
\text { nach der } \\
2 . \text { Woche }\end{array}$ & $300-600 \mathrm{mg} / \mathrm{d}$ & $\begin{array}{l}\text { Psychiatrische } \\
\text { Störungen, Ge- } \\
\text { wichtszu- oder - } \\
\text { abnahme, Ödeme, } \\
\text { selten Pankreatitis }\end{array}$ & - & - \\
\hline $\begin{array}{l}\text { Etho- } \\
\text { suximid }\end{array}$ & ESM & $\begin{array}{l}\mathrm{Ca}^{2+}-\text { Kanal- } \\
\text { blockade }\end{array}$ & $\begin{array}{l}\text { Monothe- } \\
\text { rapie gene- } \\
\text { ralisierter } \\
\text { Epilepsien } \\
\text { (Absencen, } \\
\text { Myokloni- } \\
\text { en) }\end{array}$ & $\begin{array}{l}250- \\
500 \mathrm{mg} / \mathrm{d}\end{array}$ & $\begin{array}{l}\text { um } 250 \mathrm{mg} \\
\text { pro Woche }\end{array}$ & $\begin{array}{l}1000- \\
2000 \mathrm{mg} / \mathrm{d}\end{array}$ & $\begin{array}{l}\text { Gewichtsverlust, } \\
\text { psychiatrische } \\
\text { Probleme (teil pa- } \\
\text { ranoide Störung), } \\
\text { Dyskinesien }\end{array}$ & \pm & $\begin{array}{l}\text { Nur gegen } \\
\text { Absencen wirksam }\end{array}$ \\
\hline $\begin{array}{l}\text { Pheno- } \\
\text { barbital }\end{array}$ & PB & $\begin{array}{l}\text { direkt GA- } \\
\text { BAerg }\end{array}$ & $\begin{array}{l}\text { Monothera- } \\
\text { pie fokaler } \\
\text { Anfälle, ge- } \\
\text { neralisierte } \\
\text { Epilepsie } \\
\text { (nicht wirk- } \\
\text { sam gegen } \\
\text { Absencen) }\end{array}$ & $\begin{array}{l}\text { nach } \\
\text { KG, ca. } \\
50 \mathrm{mg} / \mathrm{d}\end{array}$ & $\begin{array}{l}\text { nach KG } \\
\text { und unter } \\
\text { Spiegelkon- } \\
\text { trolle }\end{array}$ & $\begin{array}{l}50-300 \mathrm{mg} / \mathrm{d} \\
\text { (auch i. v. vorh.) }\end{array}$ & $\begin{array}{l}\text { Sedierung, Abhän- } \\
\text { gigkeit, depressive } \\
\text { Verstimmung, } \\
\text { kognitive Störung, } \\
\text { Atemdepression, } \\
\text { Dupuytren- } \\
\text { Kontrakturen, } \\
\text { Osteoporose, At- } \\
\text { tackenauslösung } \\
\text { bei Porphyrie }\end{array}$ & ++ & $\begin{array}{l}\text { Nicht mehr erste } \\
\text { Wahl, da häufige } \\
\text { Nebenwirkungen, } \\
\text { Toleranzentwick- } \\
\text { lung, Abhängig- } \\
\text { keitsrisiko und } \\
\text { hohes Interak- } \\
\text { tionspotenzial. } \\
\text { Bei Absetzen } \\
\text { hohes Risiko für } \\
\text { Entzugsanfälle. }\end{array}$ \\
\hline
\end{tabular}


> Tab. 1 Fortsetzung.

\begin{tabular}{|c|c|c|c|c|c|c|c|c|c|}
\hline Name & Code & $\begin{array}{l}\text { Mechanis- } \\
\text { mus }\end{array}$ & Indikation & $\begin{array}{l}\text { Startdosis } \\
\text { (Erwach- } \\
\text { sene) }\end{array}$ & $\begin{array}{l}\text { Steigerung } \\
\text { (Erwach- } \\
\text { sene) }\end{array}$ & $\begin{array}{l}\text { Zieldosis } \\
\text { bis max. } \\
\text { zugelassene } \\
\text { Tagesdosis } \\
\text { (Erwachsene) }\end{array}$ & $\begin{array}{l}\text { Wichtigste } \\
\text { spezifische UAW }\end{array}$ & $\begin{array}{l}\text { Interak- } \\
\text { tions- } \\
\text { poten- } \\
\text { zial }\end{array}$ & $\begin{array}{l}\text { UAW/ } \\
\text { Bemerkungen }\end{array}$ \\
\hline $\begin{array}{l}\text { Primi- } \\
\text { don }\end{array}$ & PRM & $\begin{array}{l}\text { direkt } \\
\text { GABAerg }\end{array}$ & $\begin{array}{l}\text { Monothera- } \\
\text { pie fokaler } \\
\text { Anfälle, ge- } \\
\text { neralisierte } \\
\text { Epilepsie } \\
\text { (nicht wirk- } \\
\text { sam gegen } \\
\text { Absencen) }\end{array}$ & $125 \mathrm{mg} / \mathrm{d}$ & $\begin{array}{l}\text { Aufdosie- } \\
\text { rung nach } \\
\text { Fachinfor- } \\
\text { mation }\end{array}$ & $750-1000 \mathrm{mg} / \mathrm{d}$ & wie PB & ++ & Prodrug von PB \\
\hline $\begin{array}{l}\text { Stiripen- } \\
\text { tol }\end{array}$ & STP & GABAerg & $\begin{array}{l}\text { Dravet- } \\
\text { Syndrom }\end{array}$ & $\begin{array}{l}250 \mathrm{bis} \\
500 \mathrm{mg}\end{array}$ & $\begin{array}{l}\text { um } 250- \\
500 \mathrm{mg} \text { pro } \\
\text { Woche }\end{array}$ & $50 \mathrm{mg} / \mathrm{kg} \mathrm{KG} / \mathrm{d}$ & $\begin{array}{l}\text { Gewichtsverlust, } \\
\text { psychiatrische } \\
\text { Symptome, Hy- } \\
\text { perkinesien }\end{array}$ & + & $\begin{array}{l}\text { Bei Dravet-Syn- } \\
\text { drom zugelassen, } \\
\text { Kombination mit } \\
\text { VPA und CLB }\end{array}$ \\
\hline $\begin{array}{l}\text { Peram- } \\
\text { panel }\end{array}$ & PER & $\begin{array}{l}\text { AMPA-Rezep- } \\
\text { torblockade }\end{array}$ & $\begin{array}{l}\text { Fokale und } \\
\text { genera- } \\
\text { lisierte } \\
\text { Anfälle }\end{array}$ & $2 \mathrm{mg} / \mathrm{d}$ & $\begin{array}{l}\text { alle } 1-2 \text { Wo- } \\
\text { chen um } \\
2 \mathrm{mg} / \mathrm{d}\end{array}$ & $4-12 \mathrm{mg} / \mathrm{d}$ & $\begin{array}{l}\text { Gewichtsverände- } \\
\text { rungen, psychiatri- } \\
\text { sche Symptome }\end{array}$ & $( \pm)$ & - \\
\hline $\begin{array}{l}\text { Rufin- } \\
\text { amid }\end{array}$ & RUF & Unbekannt & $\begin{array}{l}\text { Lennox- } \\
\text { Gastaut- } \\
\text { Syndrom }\end{array}$ & $\begin{array}{l}\text { bei }>30 \mathrm{~kg} \\
\text { KG: } \\
400 \mathrm{mg} / \mathrm{d}\end{array}$ & $\begin{array}{l}\text { bei }>30 \mathrm{~kg} \\
\text { KG: } z . B . \mathrm{um} \\
400 \mathrm{mg} / \mathrm{d} \\
\text { alle } 2-3 \mathrm{~d}\end{array}$ & $\begin{array}{l}1000- \\
3000 \mathrm{mg} / \mathrm{d}\end{array}$ & $\begin{array}{l}\text { Gewichtsverlust, } \\
\text { Sedierung }\end{array}$ & + & $\begin{array}{l}\text { Nur bei Lennox- } \\
\text { Gastaut-Syndrom } \\
\text { zugelassen; Dosis } \\
\text { verringern bei } \\
\text { gleichzeitiger } \\
\text { VPA-Therapie }\end{array}$ \\
\hline
\end{tabular}

AMPA: $\alpha$-Amino-3-hydroxy-5-methyl-4-isoxazolpropionsäure, BCECTS: benign childhood epilepsy with centrotemporal spikes, CLB: Clobazam, GABA: Y-Aminobuttersäure, GTKA: generalisiert (bilateral) tonisch-klonischer Anfall, HLA: humanes Leukozytenantigen, IGE: genetisch (idiopathisch) generalisierte Epilepsie, JME: juvenile myoklonische Epilepsie, KG: Körpergewicht, NMDA: N-Methyl-D-Aspartat, SV2A: synaptisches Vesikelprotein 2 A; UAW: unerwünschte Arzneimittelwirkungen

die Monotherapie und Zusatztherapie fokaler Anfälle ab dem 4. Lebensjahr. Etwa die Hälfte des Medikamentes wird unverändert renal ausgeschieden, der Rest hepatisch metabolisiert, die Halbwertszeit beträgt etwa 14 Stunden (steady state nach 3 Tagen). LCM steht zur oralen wie zur parenteralen Gabe zur Verfügung. Die erste Dosis bei Erwachsenen beträgt üblicherweise $100 \mathrm{mg} / \mathrm{d}$, verteilt auf zwei Gaben. Die Zieldosis beträgt 200-400 mg/d, maximal 600 mg/d. Add-on-Responderraten von ca. 70\% [26] und eine Ein-Jahres-Anfallsfreiheit bei Monotherapie von etwa $65 \%$ [27, 28] werden berichtet. Bei Patienten mit bekannten Herzrhythmusstörungen oder schwerer Herzerkrankung sollte vor Therapiebeginn ein EKG abgeleitet werden. Ein AV-Block 2. oder 3. Grades stellt eine Kontraindikation dar. Ein wesentlicher Vorteil ist die vorhandene i.v.-Formulierung, die eine rasche Aufsättigung auch im Notfall ermöglicht [28].

Lamotrigin (LTG) ist zur Monotherapie fokaler und generalisierter Epilepsien ab dem 13. Lebensjahr zugelassen (ab dem 2. Lebensjahr als Zusatztherapie und als Monotherapie typischer Absencen). Es hemmt, wie CBZ und PHT, spannungsabhängige Natriumkanäle über eine Verstär- kung der schnellen Inaktivierung. Die Halbwertszeit beträgt 29 Stunden, es wird hepatisch abgebaut. Während LTG selbst das CYP-System nicht beeinflusst, verstärken PHT, PB und CBZ den Abbau von LTG. VPA verlangsamt als CYP-Inhibitor den LTG-Abbau und verdoppelt deshalb im Mittel den LTG-Spiegel, sodass die Dosis ggf. anzupassen ist. Orale Kontrazeptiva können die LTG-Wirkung reduzieren, und selten umgekehrt. Die Zieldosis bei Erwachsenen beträgt 100-400 mg/d (auch höher möglich), aufgeteilt auf 2 Gaben, die Initialdosis beträgt 25 mg. Die Responderrate lag in den Zulassungsstudien bei $35 \%$. LTG ist vergleichbar wirksam wie CBZ, jedoch besser verträglich [29]. Die wichtigste Nebenwirkung von LTG sind allergische Hautreaktionen, die bis zum Stevens-Johnson-Syndrom reichen können. Das Risiko des Auftretens lässt sich durch langsame Aufdosierung verringern, bei Komedikation mit VPA sollte mit $5 \mathrm{mg}$ oder $12,5 \mathrm{mg}$ langsam begonnen werden. LTG ist ein bei fokalen Epilepsien gut wirksames (Mittel der bevorzugten ersten Wahl in den Leitlinien der Deutschen Gesellschaft für Neurologie, DGN) und meist gut verträgliches Antianfallsmedikament. Es ist eines der Mittel der Wahl bei Patientinnen mit Schwangerschaftswunsch, da das Risiko schwerwiegender kind- 
licher Fehlbildungen bei Dosen bis 300 mg/d nicht signifikant erhöht ist [22]. Auch bei älteren Patienten oder bei befürchteten kognitiven Nebenwirkungen kann es gut eingesetzt werden. Weniger gut geeignet ist es, wenn ein schneller Wirkeintritt erforderlich ist sowie bei Allergieneigung. Gegen generalisierte Anfälle, insbesondere myoklonische Anfälle und Absencen [30, 31] ist LTG schlechter wirksam und kann myoklonische Anfälle auch verstärken.

Levetiracetam (LEV) ist zur Monotherapie fokaler Epilepsien ab dem 16. Lebensjahr und zur Kombinationstherapie ab dem 1. Lebensmonat zugelassen sowie zur Zusatzbehandlung generalisierter Epilepsien ab dem 12. Lebensjahr. LEV interagiert mit dem präsynaptischen Vesikelprotein SV2A und führt dadurch zu Veränderungen des vesikulären Transportes und der Exozytose, der genaue Mechanismus der antikonvulsiven Wirkung ist jedoch unbekannt [32]. Die Halbwertszeit von LEV beträgt 7 Stunden, die Ausscheidung erfolgt überwiegend renal. Die Zieldosis bei Erwachsenen beträgt $1000-3000 \mathrm{mg} / \mathrm{d}$ verteilt auf 2 Gaben, die erste Dosis liegt zwischen $2 \times 250 \mathrm{mg} / \mathrm{d}$ und $2 \times 500 \mathrm{mg} / \mathrm{d}$. LEV ist auch intravenös und als Saft verfügbar. In der Add-on-Therapie zeigte sich eine Responderrate von $30 \%$ bis $40 \%[33,34]$ in der Monotherapie blieben $56 \%$ der Patienten über ein Jahr anfallsfrei [16]. Spezifische UAW sind Reizbarkeit, Aggressivität und Depression. Das Risiko schwerwiegender kindlicher Fehlbildungen ist bei LEV-Einnahme in der Schwangerschaft nicht signifikant erhöht [22]. LEV ist bei fokalen und generalisierten Epilepsien gut wirksam. Es besitzt keine nennenswerten Arzneimittelinteraktionen, auch die Verträglichkeit bei älteren Patienten ist sehr gut [35]. Die Leitlinie der DGN nennt LEV als Mittel der bevorzugten ersten Wahl bei fokalen Epilepsien, und dort wird es bei $60 \%$ der Neueinstellungen verwendet $[2,36]$. Bei bekannter Depression ist es nur vorsichtig einzusetzen, bei Niereninsuffzienz muss die Dosis gegebenenfalls angepasst werden.

Brivaracetam (BRV) teilt den Wirkmechanismus von LEV, bindet jedoch mit einer höheren Affinität an das SV2A-Protein. BRV ist zur Zusatzbehandlung fokaler Anfälle ab dem 4. Lebensjahr zugelassen. Die Therapie bei Erwachsenen sollte mit $50 \mathrm{mg} / \mathrm{d}$ begonnen werden, die Zieldosis beträgt 100-200 mg/d, verteilt auf zwei Gaben. Die Halbwertszeit beträgt 7-8Stunden, die Elimination erfolgt vorwiegend renal. Auch BRV ist zur intravenösen Gabe und als Saft erhältlich. In der Add-on-Therapie sind Responderraten von $30 \%$ bis $40 \%$ beschrieben [37]. Gegenüber LEV weist BRV wahrscheinlich ein geringeres Risiko für psychobehaviorale Nebenwirkungen auf [38], die Wirksamkeit ist vergleichbar. Eine Umstellung von Levetiracetam auf BRV kann in einem Verhältnis von 10:1 direkt über Nacht erfolgen [38]. Es gibt positive Hinweis für die Wirksamkeit auch bei genetisch generalisierten Epilepsien [39] (in Deutschland ist BRV nicht für diese Indikation zugelassen).
Valproat (VPA) hemmt Natriumkanäle und modifiziert den zerebralen GABA-Stoffwechsel [40, 41], der tatsächliche Beitrag dieser Effekte zur antikonvulsiven Wirkung ist jedoch nicht genau bekannt. Es ist zugelassen zur Behandlung fokaler und generalisierter Epilepsien bei Kindern und Erwachsenen. Die erste Dosis bei Erwachsenen beträgt $300 \mathrm{mg} / \mathrm{d}$, die Zieldosis 600-1500 mg/d und kann in Einzelfällen bis $2000 \mathrm{mg} / \mathrm{d}$ auftitriert werden. VPA ist ein potenter CYP-Inhibitor und interagiert mit vielen Antianfallsmedikamenten. Eine VPA-Spiegelkontrolle ist regelmäßig notwendig. Valproat war in der SANAD Vergleichsstudie das wirksamste Medikament bei generalisierten Epilepsien [18]. Bei Frauen im gebärfähigen Alter darf es nicht eingesetzt werden, da ein sehr hohes Risiko für kindliche Fehlbildungen von bis zu $25 \%$ besteht [22]. Nur wenn medikamentöse Alternativen nicht verfügbar oder verträglich sind, kann es unter strengen Auflagen (z. B. regelmäBige jährliche schriftliche Einverständnis und Aufklärung nach Vorgaben des BfArM, regelmäßige Kontrolle der Indikation, sichere Verhütungsmethode) bei Mädchen und Frauen im gebärfähigen Alter angewendet werden. Häufige unerwünschte Arzneimittelwirkungen (UAW) sind eine Gewichtszunahme, Tremor und Haarausfall. In der Langzeittherapie kann VPA Osteoporose begünstigen.

Zonisamid (ZNS) ist zugelassen zur Monotherapie fokaler Epilepsien bei Erwachsenen mit neu diagnostizierter Epilepsie sowie als Zusatztherapie fokaler Epilepsien ab dem 6. Lebensjahr. Es wirkt primär über Hemmung von Natrium- und T-Typ-Kalziumkanälen. Die Halbwertszeit beträgt 60 Stunden (steady state nach 8-14 Tagen), die Metabolisierung erfolgt überwiegend hepatisch. Die Zieldosis beträgt $300-500 \mathrm{mg} / \mathrm{d}$, die Initialdosis beträgt meist $2 \times 25 \mathrm{mg} / \mathrm{d}$. In der Add-on-Therapie fokaler Epilepsien ergaben sich für ZNS Responderraten von $30 \%$ [42]. Es gibt positive Hinweis für die Wirksamkeit auch bei genetisch generalisierten Epilepsien [43] (in Deutschland ist ZNS nicht für diese Indikation zugelassen). Wichtige UAW sind kognitive Einschränkungen, Gewichtsverlust sowie das Auftreten von Nierensteinen. Eine Kombination mit anderen Carboanhydrasehemmern (auch Topiramat) wird aufgrund der Addition der Nebenwirkungen (Parästhesien, Nierensteine) nicht empfohlen.

Topiramat (TPM) ist zugelassen für die Monotherapie fokaler und generalisierter Epilepsien ab dem 6. Lebensjahr (ab dem 2. Lebensjahr als Zusatztherapie). Es wirkt über multiple Mechanismen antikonvulsiv, u. a. hemmt es Glutamatrezeptoren und die Carboanhydrase, erhöht den GABAbedingten Chloridinflux in Nervenzellen und blockiert spannungsabhängige Natriumkanäle. Seine Halbwertszeit beträgt 20-30 Stunden (steady state nach 4-8 Tage), die Ausscheidung erfolgt vor allem renal. CBZ und PHT reduzieren die TPM-Halbwertszeit um $50 \%$. Die zugelassene Dosis beträgt $50-500 \mathrm{mg} / \mathrm{d}$, verteilt auf 2 Gaben, selten werden jedoch mehr als 300 mg/d im klinischen Alltag benötigt. Die erste Dosis beträgt üblicherweise $25 \mathrm{mg} / \mathrm{d}$. Die 
Responder-Rate in der Add-on-Therapie liegt bei $35 \%$ bis $50 \%$ [44], eine 12-monatige Anfallsfreiheit fand sich bei $60 \%$ bis $75 \%$ der Patienten unter TPM-Monotherapie [45]. Relevante Nebenwirkungen sind dosisabhängige, reversible kognitive Einschränkungen inklusive Sprachstörungen (gut und schnell messbar in 5-10 Minuten im klinischen Alltag mit kurzen neuropsychologischen Tests, wie dem EpiTrack), akrale und orale Parästhesien sowie die Entwicklung von Nierensteinen. TPM zeigte in einer rezenten Auswertung der EURAP-Datenbank bei einer kleinen Fallzahl ein gering erhöhtes Fehlbildungsrisiko in der Monotherapie [22], es gibt jedoch Hinweise auf eine intrauterine Wachstumsverzögerung. TPM ist ein sehr wirksames Antianfallsmedikament und kann bei allen Epilepsieformen eingesetzt werden, jedoch können unerwünschte Arzneimittelwirkungen wie Denkverlangsamung und Wortfindungsstörungen therapielimitierend sein (v. a. bei Tagesdosen über $100 \mathrm{mg}$ ).

Gabapentin (GBP) ist zur Therapie von fokalen Epilepsien zugelassen. Es wirkt auf präsynaptische Kalziumkanäle und besitzt nur wenige Arzneimittelinteraktionen. Seine antikonvulsive Wirksamkeit ist eher gering (Addon-Responderrate $25 \%$ ) [46], die Wirksamkeit war in der SANAD-Studie geringer als die von CBZ und LTG. Aufgrund der guten Verträglichkeit kann es bei älteren Patienten eingesetzt werden, nachteilig ist jedoch die aufgrund der kurzen Halbwertzeit erforderliche tägliche Dreimalgabe.

Pregabalin (PGB) wirkt ebenso wie Gabapentin als Hemmer von präsynaptischen Kalziumkanälen und hat wie dieses, trotz des Namens, keine direkte GABAerge Wirkung. PGB ist zur Zusatztherapie fokaler Epilepsien zugelassen, zudem bei neuropathischen Schmerzen und generalisierter Angststörung. Es hat eine Halbwertszeit von 5-6Stunden, eine relevante Metabolisierung findet nicht statt. PGB wird überwiegend unverändert renal ausgeschieden, weswegen die Dosis bei Niereninsuffizienz ggf. anzupassen ist. Es besitzt nur wenige Arzneimittelinteraktionen. In Add-on-Therapiestudien zeigte sich eine Responderrate von $40 \%$ bis $50 \%$ [47], in einer direkten Monotherapievergleichsstudie war es jedoch LTG unterlegen [48].

Perampanel (PER) wirkt als AMPA-Rezeptorantagonist. Es ist das erste Antianfallsmedikament mit diesem Wirkmechanismus auf dem deutschen Markt. PER ist zugelassen für die Zusatztherapie fokaler Epilepsien und generalisierter Epilepsien bei Vorliegen primär generalisierter (bilateral) tonisch-klonischer Anfälle, jeweils ab dem 12. Lebensjahr. Die Halbwertszeit ist mit 105 Stunden recht lang, der steady state wird nach 2-3 Wochen erreicht [49]. PER wird vor allem über das Cytochrom P450(CYP)-System metabolisiert und unterliegt damit einer Interaktion mit den Antianfallsmedikamenten CBZ, OXC, PHT und TPM (erhöhte PER-Metabolisierung). VPA, LEV und ZNS scheinen keinen relevanten Einfluss auf die PER-Clearance zu haben. Bei Komedikation zu OXC kann die Serumkonzentration von OXC um bis zu $30 \%$ steigen [50]. PER kann nur oral als Tablette und Suspension verabreicht werden. Die Therapie bei Erwachsenen wird üblicherweise mit $2 \mathrm{mg} / \mathrm{d}$ begonnen, die erste Zieldosis liegt bei $4-8 \mathrm{mg} / \mathrm{d}$ (maximal $12 \mathrm{mg} / \mathrm{d}$ ). In der Add-on-Therapie werden Responderraten von $30 \%$ bis 40 \% für fokale Anfälle und 64 \% für bilateral tonisch-klonische Anfälle erreicht [51]. Ein Vorteil, auch in Bezug auf mögliche Nebenwirkungen, wie Schwindel und Müdigkeit, ist die einmal tägliche Einnahme abends, am besten unmittelbar vor dem Zubettgehen. Ebenfalls auftreten kann Aggression [52]. Die Einsatzmöglichkeit bei generalisierten Epilepsien sowie der neue Wirkmechanismus stellen einen Therapiefortschritt dar. Die mitunter therapielimitierenden Nebenwirkungen können durch Wahl des Einnahmezeitpunktes und langsame Eindosierung abgemildert werden.

Die Antianfallsmedikamente Vigabatrin, Rufinamid, Felbamat und Stiripentol sind nur für spezielle Epilepsiesyndrome oder als Reservemedikamente zugelassen, wir verweisen hier auf andere Übersichtsstudien [53-57].

Cannabidiol (CBD) ist ein antikonvulsiv wirksames Cannabinoid, welches jedoch nicht maßgeblich auf die Endocannabinoidrezeptoren CB1 und CB2 wirkt und keinen psychotropen Effekt aufweist. Der genaue antikonvulsive Mechanismus ist noch unbekannt. CBD zeigte in prädefinierter Dosierung von 10 bis 20 mg/kg Körpergewicht in 2 Studien eine moderate Wirksamkeit bei bestimmten Anfällen beim Lennox-Gastaut- [58] und Dravet-Syndrom [59]. Belastbare Daten in der Behandlung anderer Epilepsieformen oder bei Erwachsenen fehlen noch. CBD ist in den USA bereits durch die FDA zugelassen. Eine Zulassung in Europa durch die EMA wird in den nächsten 12 Monaten erwartet. Ein antikonvulsiver Effekt von Tetrahydrocannabinol (THC) konnte nicht nachgewiesen werden [60].

\section{Akuttherapie von epileptischen Anfällen, Anfallsserien und Status epilepticus}

Epileptische Anfälle sind in der Regel selbstlimitierend und dauern im Durchschnitt etwa 2 Minuten [61]. Prolongierte epileptische Anfälle ab einer Dauer von 5 Minuten bei generalisiert tonisch-klonischen Anfällen beziehungsweise ab einer Dauer von 10 Minuten bei fokalen Anfällen oder Absencen werden nach den neuesten Empfehlungen der ILAE (International League Against Epilepsy) als Status epilepticus definiert und sollten zu diesem Zeitpunkt behandelt werden [62], da ein längerer Status mit einer erhöhten Mortalität assoziiert ist [63, 64]. Dabei erfolgt die Ersttherapie immer mit Benzodiazepinen, wobei verschiedene Darreichungsformen und Wirkstoffe zur Verfügung stehen. 
- Tab. 2 Übersicht über intravenöse Benzodiazepine (nach Daten aus [70, 71]).

\begin{tabular}{|l|l|l|l|l|}
\hline & Midazolam & Lorazepam & Clonazepam & Diazepam \\
\hline Initialdosis (mg) beim Erwachsenen ${ }^{2}$ & $\begin{array}{l}5-10, \text { fraktioniert in 2- } \\
\text { bis 3-mg-Schritten }\end{array}$ & $2-4$ & 1 & 10 \\
\hline Empfohlene intravenöse Dosierung (mg/kg KG) & 0,1 & $0,05-0,1$ & 0,015 & 0,15 \\
\hline Maximaldosis (mg) & 20 & 8 & 3 & 30 \\
\hline Halbwertszeit (Stunden) & $3-4$ & $12-16$ & $30-40$ & $20-100^{2}$ \\
\hline Interaktionen & wenige & wenige & viele & viele \\
\hline Gewebetoxizität & gering & gering & gering & gering \\
\hline
\end{tabular}

KG: Körpergewicht; ' $U m$ eine Unterdosierung zu vermeiden, ist die erneute Gabe von Lorazepam und Diazepam über die typische Initialdosis (entspricht 1-2 Ampullen) hinaus vorzunehmen (CAVE: Atemdepression). ${ }^{2}$ aufgrund der schnellen Umverteilung liegt eine kurze Wirkdauer im ZNS vor

- Tab. 3 Übersicht über nicht intravenöse Benzodiazepine

\begin{tabular}{|l|l|l|l|l|}
\hline & Midazolam bukkal & Midazolam intranasal & Midazolam intramuskulär & Diazepam rektal \\
\hline Initialdosis beim Erwachsenen (mg) & 10 & $5-10$ & 10 & $5-10$ \\
\hline Empfohlene Dosierung (mg/kg KG) & 0,2 & 0,2 & $0,05-0,15$ & 0,2 \\
\hline Maximaldosis (mg) & 10 & 15 & 15 & 30 \\
\hline Nebenwirkungen, Besonderheiten & Vergleichsweise teuer & $\begin{array}{l}\text { Nasale Irritationen, muss in } \\
\text { Apotheke angefertigt werden }\end{array}$ & Schmerzhafte Injektion & $\begin{array}{l}\text { Soziale } \\
\text { Stigmatisierung }\end{array}$ \\
\cline { 2 - 5 } & & &
\end{tabular}

In den Leitlinien der DGN zum Status epilepticus [65] wird als Mittel der Wahl Lorazepam i. v. in einer Dosierung von 0,05 bis $0,1 \mathrm{mg}$ pro Kilogramm Körpergewicht (KG) bei Gabe durch einen Arzt oder bis zu einer Gesamtdosis von 2 bis $4 \mathrm{mg}$ bei Gabe durch einen Rettungsassistenten empfohlen. Lorazepam hat im Vergleich zu anderen Benzodiazepinen eine relativ lange intrazerebrale Halbwertszeit ( Tab. 2) und hat sich in Studien als gut verträglich und wirksam herausgestellt $[66,67]$. In der Schweiz und in Frankreich wird als Mittel der Wahl Clonazepam als langsame Bolusinjektion in einer Dosierung von $0,015 \mathrm{mg} / \mathrm{kg}$ KG empfohlen, welches ebenfalls eine relativ lange intrazerebrale Halbwertszeit aufweist. In einer multizentrischen prospektiven Beobachtungsstudie zur Behandlung des Status epilepticus in deutschsprachigen Ländern (SENSE-Register) zeigte sich jedoch, dass Lorazepam in der Initialtherapie häufiger unterdosiert verabreicht wurde als Clonazepam und somit mit einem schlechteren Outcome des SE vergesellschaftet war $[68,69]$. Neben Lorazepam und Clonazepam können auch noch andere Benzodiazepine intravenös eingesetzt werden ( $\triangleright$ Tab. 2).

Neben der intravenösen Darreichungsform stehen noch andere Applikationswege für die Verabreichung von Benzodiazepinen zur Verfügung, welche insbesondere außerhalb des Krankenhauses in der Initialtherapie durch medizinische Laien Anwendung finden. Die beste Evidenz existiert dabei bislang für die Gabe von intramuskulärem Midazo- lam, welches in einer randomisierten kontrollierten Studie zu einer signifikant höheren Anfallsunterbrechungsrate im Vergleich zur Gabe von Lorazepam i. v. geführt hat. Die Autoren schlussfolgerten, dass dies in der kürzeren Zeit bis zur Applikation begründet lag, da die Etablierung eines intravenösen Zuganges lange dauern kann [72]. Die längste Erfahrung besteht für Diazepam-Rektiolen, welche seit den 1990er-Jahren als Standardtherapie zur Anfallsdurchbrechung etabliert sind. Auch hierfür wurden in mehreren Studien die Sicherheit und Wirksamkeit bestätigt [73]. Problematisch ist jedoch die soziale Stigmatisierung aufgrund der rektalen Verabreichung. In Studien konnte zudem die Gleichwertigkeit oder Überlegenheit von intranasalen und bukkalen Applikationen von Midazolam oder Lorazepam im Vergleich zu rektalem Diazepam bzw. intravenösem Lorazepam gezeigt werden [74-76]. In Deutschland steht bukkales Midazolam als verschreibungsfähiges Handelspräparat mit einer Zulassung für Kinder und Jugendliche (<3 Monate bis < 18 jahre) zur Verfügung. Intranasales Midazolam wird in Deutschland im Video-EEG-Monitoring regelhaft eingesetzt und kann in Apotheken auf Rezept angefertigt werden. Entsprechende Rezept- und Herstellungsdetails sind in der Studie von Kay et al. veröffentlicht worden [77]. Aufgrund der langen Resorptionshalbwertszeit oral verfügbarer Benzodiazepine von etwa 20 Minuten sind diese in der Akuttherapie epileptischer Anfälle nicht zu empfehlen (Lorazepam sublingual, Clonazepam oral, Diazepam oral), werden klinisch jedoch oft verordnet [78] ( Tab.3). 


\section{FAZIT FÜR DIE PRAXIS}

Fokale Epilepsien können mit fast allen Antianfallsmedikamenten behandelt werden. Eine Wirküberlegenheit eines Medikamentes ist - mit wenigen Ausnahmen - meist nicht belegt. Deswegen sollte die Therapie mit dem Patienten hinsichtlich des Nebenwirkungsprofils, der Begleiterkrankungen, der Komedikation und sozialer Aspekte (Schwangerschaftswunsch!) ausgewählt werden. In der Regel werden Levetiracetam oder Lamotrigin zur initialen Therapie verwendet. Auch bei genetischen generalisierten Epilepsien (GGE) sind diese Faktoren zu bedenken. Hier kommen Levetiracetam, Lamotrigin, Valproat, Ethosuximid, Topiramat, Perampanel und (off label, aber mit guten Daten aus Japan und den USA) Zonisamid infrage. Natriumkanalblocker wie Carbamazepin oder Medikamente wie Gabapentin oder Pregabalin können bei GGE prokonvulsiv wirken und sogar einen Status epilepticus auslösen. Neuere Antianfallsmedikamente zeigten keine Wirküberlegenheit, sind aber meist besser verträglich. Dem gegenüber stehen höhere Tagestherapiekosten der meisten neueren Medikamente [36, 79]. Sie erweitern aber das Spektrum der individuell einsetzbaren Antianfallsmedikamente und damit die Chance einer gut verträglichen und individuell erfolgreichen Pharmakotherapie.

Bei prolongierten epileptischen Anfällen bedarf es einer schnellen und ausreichend hoch dosierten Gabe von Benzodiazepinen, um so einen therapierefraktären Verlauf abwenden zu können und zu einem besseren Outcome beizutragen. Um Therapieverzögerungen zu vermeiden, sollten insbesondere nicht intravenöse Darreichungsformen berücksichtigt werden. Randomisierte kontrollierte Studien zum Vergleich der unterschiedlich verabreichten Benzodiazepine sind weiterhin ausstehend.

\section{Interessenkonflikt}

\section{Erklärung zu finanziellen Interessen}

Forschungsförderung erhalten: ja; Honorar/geldwerten Vorteil für Referententätigkeit erhalten: ja; Bezahlter Berater/interner Schulungsreferent/Gehaltsempfänger: ja; Patent/Geschäftsanteile/Aktien (Autor/Partner, Ehepartner, Kinder) an Firma: nein.

Erklärung zu nicht finanziellen Interessen

Prof. A. Strzelczyk ist Vorstandsmitglied der Deutschen Gesellschaft für Epileptologie (DGfE) und Sekretär der Arbeitsgemeinschaft für prächirurgische Diagnostik und operative Epilepsietherapie.

\section{Korrespondenzadresse}

Prof. Dr. med. Adam Strzelczyk, MHBA

Epilepsiezentrum Frankfurt Rhein-Main

Zentrum der Neurologie und Neurochirurgie

Goethe-Universität Frankfurt

Schleusenweg 2-16, Haus 95, 60528 Frankfurt am Main

Tel. 069/63017466, Fax 069/630184466

strzelczyk@med.uni-frankfurt.de

\section{Verantwortlicher ärztlicher Herausgeber}

Wissenschaftlich verantwortlich gemäß Zertifizierungsbedingungen für diesen Beitrag ist Prof. Dr. med. Adam Strzelczyk, Frankfurt am Main.

\section{Literatur}

[1] Forsgren L, Beghi E, Oun A et al. The epidemiology of epilepsy in Europe - a systematic review. Eur J Neurol 2005; 12(4): 245-53

[2] Ertl J, Hapfelmeier J, Peckmann T et al. Guideline conform initial monotherapy increases in patients with focal epilepsy: A population-based study on German health insurance data. Seizure 2016; 41: 9-15

[3] Hamer HM, Dodel R, Strzelczyk A et al. Prevalence, utilization, and costs of antiepileptic drugs for epilepsy in Germany - a nationwide population-based study in children and adults. Journal of neurology 2012; 259(11): 2376-84

[4] Fisher RS, Acevedo C, Arzimanoglou A et al. ILAE Official Report: A practical clinical definition of epilepsy. Epilepsia 2014; 55(4): 475-82

[5] Strzelczyk A, Griebel C, Lux W et al. The Burden of Severely Drug-Refractory Epilepsy: A Comparative Longitudinal Evaluation of Mortality, Morbidity, Resource Use, and Cost Using German Health Insurance Data. Frontiers in neurology 2017; 8: 712

[6] Willems LM, Richter S, Watermann N et al. Trends in resource utilization and prescription of anticonvulsants for patients with active epilepsy in Germany from 2003 to 2013 - A tenyear overview. Epilepsy Behav 2018; 83: 28-35

[7] Kwan P, Schachter SC, Brodie MJ. Drug-resistant epilepsy. The New England journal of medicine 2011; 365(10): 919-26

[8] Kwan P, Arzimanoglou A, Berg AT et al. Definition of drug resistant epilepsy: consensus proposal by the ad hoc Task Force of the ILAE Commission on Therapeutic Strategies. Epilepsia 2010; 51(6): 1069-77

[9] Rosenow F, Bast T, Czech T et al. Revised version of quality guidelines for presurgical epilepsy evaluation and surgical epilepsy therapy issued by the Austrian, German, and Swiss working group on presurgical epilepsy diagnosis and operative epilepsy treatment. Epilepsia 2016; 57(8): 1215-20

[10] Li-Na Z, Deng C, et al. Indirect comparison of third-generation antiepileptic drugs as adjunctive treatment for uncontrolled focal epilepsy. Epilepsy Res 2018; 139: 60-72

[11] Bodalia PN, Grosso AM, Sofat R et al. Comparative efficacy and tolerability of anti-epileptic drugs for refractory focal epilepsy: systematic review and network meta-analysis reveals 
the need for long term comparator trials. Br J Clin Pharmacol 2013; 76(5): 649-67

[12] Zhuo C, Jiang R, Li G et al. Efficacy and Tolerability of Second and Third Generation Anti-epileptic Drugs in Refractory Epilepsy: A Network Meta-Analysis. Sci Rep 2017; 7(1): 2535

[13] Otoul C, Arrigo C, et al. Meta-analysis and indirect comparisons of levetiracetam with other second-generation antiepileptic drugs in partial epilepsy. Clin Neuropharmacol 2005; 28(2): 72-8

[14] Brodie M], Sills G]. Combining antiepileptic drugs--rational polytherapy? Seizure 2011; 20(5): 369-75

[15] Marson AG, Al-Kharusi AM, Alwaidh M et al. The SANAD study of effectiveness of carbamazepine, gabapentin, lamotrigine, oxcarbazepine, or topiramate for treatment of partial epilepsy: an unblinded randomised controlled trial. Lancet 2007; 369 (9566): 1000-15

[16] Brodie M], Perucca E, Ryvlin P et al. Comparison of levetiracetam and controlled-release carbamazepine in newly diagnosed epilepsy. Neurology 2007; 68 (6): 402-8

[17] Baulac M, Brodie M], Patten A et al. Efficacy and tolerability of zonisamide versus controlled-release carbamazepine for newly diagnosed partial epilepsy: a phase 3 , randomised, double-blind, non-inferiority trial. Lancet Neurol 2012; 11 (7): 579-88

[18] Marson AG, Al-Kharusi AM, Alwaidh M et al. The SANAD study of effectiveness of valproate, lamotrigine, or topiramate for generalised and unclassifiable epilepsy: an unblinded randomised controlled trial. Lancet 2007; 369 (9566): 1016-26

[19] Glauser T, Ben-Menachem E, et al. Updated ILAE evidence review of antiepileptic drug efficacy and effectiveness as initial monotherapy for epileptic seizures and syndromes. Epilepsia 2013; 54 (3): 551-63

[20] Kwan P, Brodie M]. Clinical trials of antiepileptic medications in newly diagnosed patients with epilepsy. Neurology 2003; 60 (11 Suppl 4): S2-12

[21] Christe W, Kramer G, Vigonius U et al. A double-blind controlled clinical trial: oxcarbazepine versus sodium valproate in adults with newly diagnosed epilepsy. Epilepsy Res 1997; 26(3): 451-60

[22] Tomson T, Battino D, et al. Comparative risk of major congenital malformations with eight different antiepileptic drugs: a prospective cohort study of the EURAP registry. Lancet Neurol 2018; 17(6): 530-8

[23] Intravooth T, Staack AM, Juerges K et al. Antiepileptic drugs-induced hyponatremia: Review and analysis of 560 hospitalized patients. Epilepsy research 2018; 143: 7-10

[24] Doeser A, Dickhof G, Reitze M et al. Targeting pharmacoresistant epilepsy and epileptogenesis with a dual-purpose antiepileptic drug. Brain 2015; 138 (Pt 2): 371-87

[25] Willems LM, Zöllner JP, Paule E et al. Eslicarbazepine acetate in epilepsies with focal and secondary generalised seizures: systematic review of current evidence. Expert review of clinical pharmacology 2018; 11(3): 309-24

[26] Zadeh WW, Escartin A, et al. Efficacy and safety of lacosamide as first add-on or later adjunctive treatment for uncontrolled partial-onset seizures: A multicentre open-label trial. Seizure 2015; 31: 72-9

[27] Giraldez BG, Toledano R, Garcia-Morales I et al. Long-term efficacy and safety of lacosamide monotherapy in the treatment of partial-onset seizures: A multicenter evaluation. Seizure 2015; 29: 119-22

[28] Bauer S, Willems LM, Paule E et al. The efficacy of lacosamide as monotherapy and adjunctive therapy in focal epilepsy and its use in status epilepticus: clinical trial evidence and experience. Ther Adv Neurol Disord 2017; 10(2): 103-26

[29] Brodie M], Richens A, Yuen AW. Double-blind comparison of lamotrigine and carbamazepine in newly diagnosed epilepsy. UK Lamotrigine/Carbamazepine Monotherapy Trial Group. Lancet 1995; 345(8948): 476-9

[30] Guerrini R, Dravet C, Genton P et al. Lamotrigine and seizure aggravation in severe myoclonic epilepsy. Epilepsia 1998; 39(5): 508-12

[31] Glauser TA, Cnaan A, et al. Ethosuximide, valproic acid, and lamotrigine in childhood absence epilepsy. N Engl J Med 2010; 362(9): 790-9

[32] Löscher W, Gillard M, Sands ZA et al. Synaptic Vesicle Glycoprotein 2A Ligands in the Treatment of Epilepsy and Beyond. CNS Drugs 2016; 30(11): 1055-77

[33] Lambrechts DA, Sadzot B, van Paesschen W et al. Efficacy and safety of levetiracetam in clinical practice: results of the SKATE trial from Belgium and The Netherlands. Seizure 2006; 15 (6): 434-42

[34] Meencke HJ, Buyle S. Assessment of a dose-response relationship of levetiracetam. Eur J Neurol 2006; 13 (9): 942-6

[35] Werhahn KJ, Trinka E, Dobesberger ] et al. A randomized, double-blind comparison of antiepileptic drug treatment in the elderly with new-onset focal epilepsy. Epilepsia 2015; 56(3): 450-9

[36] Willems LM, Hamer HM, Knake S et al. General Trends in Prices and Prescription Patterns of Anticonvulsants in Germany between 2000 and 2017: Analysis of National and Cohort-Based Data. Appl Health Econ Health Policy 2019. doi:10.1007/s40258-019-00487-2

[37] Strzelczyk A, Klein KM, Willems LM et al. Brivaracetam in the treatment of focal and idiopathic generalized epilepsies and of status epilepticus. Expert review of clinical pharmacology 2016; 9(5): 637-45

[38] Steinig I, von Podewils F, Möddel G et al. Postmarketing experience with brivaracetam in the treatment of epilepsies: A multicenter cohort study from Germany. Epilepsia 2017; 58(7): 1208-16

[39] Strzelczyk A, Kay L, Bauer S et al. Use of brivaracetam in genetic generalized epilepsies and for acute, intravenous treatment of absence status epilepticus. Epilepsia 2018; 59 (8): 1549-56

[40] Löscher W. Basic pharmacology of valproate: a review after 35 years of clinical use for the treatment of epilepsy. CNS Drugs 2002; 16 (10): 669-94

[41] McLean MJ, Macdonald RL. Sodium valproate, but not ethosuximide, produces use- and voltage-dependent limitation of high frequency repetitive firing of action potentials of mouse central neurons in cell culture. J Pharmacol Exp Ther 1986; 237 (3): 1001-11

[42] Schmidt D, Jacob R, Loiseau P et al. Zonisamide for add-on treatment of refractory partial epilepsy: a European doubleblind trial. Epilepsy Res 1993; 15(1): 67-77

[43] Marinas A, Villanueva V, Giraldez BG et al. Efficacy and tolerability of zonisamide in idiopathic generalized epilepsy. Epileptic Disord 2009; 11 (1): 61-6

[44] Vajda FJ. New antiepileptic drugs. J Clin Neurosci 2000; 7 (2): 88-101

[45] Arroyo S, Dodson WE, Privitera MD et al. Randomized dose-controlled study of topiramate as first-line therapy in epilepsy. Acta Neurol Scand 2005; 112 (4): 214-22 
[46] Panebianco M, Al-Bachari S, Weston J et al. Gabapentin add-on treatment for drug-resistant focal epilepsy. Cochrane Database Syst Rev 2018; 10: CD001415

[47] Beydoun A, Uthman BM, Kugler AR et al. Safety and efficacy of two pregabalin regimens for add-on treatment of partial epilepsy. Neurology 2005; 64 (3): 475-80

[48] Kwan P, Brodie M], Kalviainen R et al. Efficacy and safety of pregabalin versus lamotrigine in patients with newly diagnosed partial seizures: a phase 3, double-blind, randomised, parallel-group trial. Lancet Neurol 2011; 10 (10): 881-90

[49] Strzelczyk A, Willems LM, Willig S et al. Perampanel in the treatment of focal and idiopathic generalized epilepsies and of status epilepticus. Expert review of clinical pharmacology 2015; 8 (6): 733-40

[50] Patsalos PN. The clinical pharmacology profile of the new antiepileptic drug perampanel: A novel noncompetitive AMPA receptor antagonist. Epilepsia 2015; 56 (1): 12-27

[51] Tsai J], Wu T, Leung $\mathrm{H}$ et al. Perampanel, an AMPA receptor antagonist: From clinical research to practice in clinical settings. Acta Neurol Scand 2018; 137 (4): 378-91

[52] Juhl S, Rubboli G. Perampanel as add-on treatment in refractory focal epilepsy. The Dianalund experience. Acta Neurol Scand 2016; 134 (5): 374-7

[53] Chiron C, Dulac O. Epilepsy: Vigabatrin treatment and visual field loss. Nat Rev Neurol 2011; 7(4): 189-90

[54] Coppola G, Besag F, et al. Current role of rufinamide in the treatment of childhood epilepsy: literature review and treatment guidelines. Eur J Paediatr Neurol 2014; 18(6): 685-90

[55] Pellock JM, Faught E, et al. Felbamate: consensus of current clinical experience. Epilepsy Res 2006; 71(2-3): 89-101

[56] Chiron C. Stiripentol for the treatment of seizures associated with Dravet syndrome. Expert Rev Neurother 2019; 19(4): $301-10$

[57] Strzelczyk A, Kortland LM, Knake S et al. Stiripentol for the treatment of super-refractory status epilepticus. Acta Neurol Scand 2015; 132(6): 435-9

[58] Devinsky O, Patel AD, Cross JH et al. Effect of Cannabidiol on Drop Seizures in the Lennox-Gastaut Syndrome. The New England journal of medicine 2018; 378 (20): 1888-97

[59] Devinsky O, Cross JH, et al. Trial of Cannabidiol for Drug-Resistant Seizures in the Dravet Syndrome. N Engl J Med 2017; 376 (21): 2011-20

[60] O'Connell BK, Gloss D, Devinsky O. Cannabinoids in treatment-resistant epilepsy: A review. Epilepsy Behav 2017; 70(Pt B): 341-8

[61] Jenssen S, Gracely EJ, Sperling MR. How long do most seizures last? A systematic comparison of seizures recorded in the epilepsy monitoring unit. Epilepsia 2006; 47(9): 1499-503

[62] Trinka E, Cock H, et al. A definition and classification of status epilepticus--Report of the ILAE Task Force on Classification of Status Epilepticus. Epilepsia 2015; 56(10): 1515-23

[63] Strzelczyk A, Ansorge S, et al. Costs, length of stay, and mortality of super-refractory status epilepticus: A population-based study from Germany. Epilepsia 2017; 58(9): 1533-41

[64] Schubert-Bast S, Zöllner JP, Ansorge S et al. Burden and epidemiology of status epilepticus in infants, children, and adolescents: A population-based study on German health insurance data. Epilepsia 2019; 60 (5): 911-20

[65] Diener HC. Leitlinien für Diagnostik und Therapie in der Neurologie: Herausgegeben von der Kommission „Leitlinien“ der DGN. Berlin 2012
[66] Prasad M, Krishnan PR, Sequeira R et al. Anticonvulsant therapy for status epilepticus. The Cochrane database of systematic reviews 2014 (9): CD003723

[67] Alldredge BK, Gelb AM, Isaacs SM et al. A comparison of lorazepam, diazepam, and placebo for the treatment of out-of-hospital status epilepticus. The New England journal of medicine 2001; 345(9): 631-7

[68] Kellinghaus C, Rossetti AO, Trinka E et al. SENSE registry for status epilepticus. Epilepsia 2018; 59 Suppl 2: 150-4

[69] Kellinghaus C, Rossetti AO, Trinka E et al. Factors predicting cessation of status epilepticus in clinical practice: Data from a prospective observational registry (SENSE). Annals of neurology 2019; 85 (3): 421-32

[70] Strzelczyk A, Kay L, Kellinghaus C et al. Concepts for Prehospital and Initial In-hospital Therapy of Status Epilepticus. Neurology International Open 2017; 01 (03): E217-E23

[71] Schubert-Bast S, Strzelczyk A. Therapie des akuten konvulsiven Anfalls und Status epilepticus im Kindesalter. Zeitschrift für Epileptologie 2019; 32 (2): 116-25

[72] Silbergleit R, Durkalski V, Lowenstein D et al. Intramuscular versus intravenous therapy for prehospital status epilepticus. The New England journal of medicine 2012; 366 (7): 591-600

[73] Dreifuss FE, Rosman NP, Cloyd JC et al. A comparison of rectal diazepam gel and placebo for acute repetitive seizures. $\mathrm{N}$ Engl J Med 1998; 338 (26): 1869-75

[74] McIntyre J, Robertson S, Norris E et al. Safety and efficacy of buccal midazolam versus rectal diazepam for emergency treatment of seizures in children: a randomised controlled trial. Lancet 2005; 366 (9481): 205-10

[75] Lahat E, Goldman M, Barr J et al. Comparison of intranasal midazolam with intravenous diazepam for treating febrile seizures in children: prospective randomised study. Bmj 2000; 321 (7253): 83-6

[76] de Haan G], van der Geest P, Doelman G et al. A comparison of midazolam nasal spray and diazepam rectal solution for the residential treatment of seizure exacerbations. Epilepsia 2010; 51 (3): 478-82

[77] Kay L, Reif PS, Belke M et al. Intranasal midazolam during presurgical epilepsy monitoring is well tolerated, delays seizure recurrence, and protects from generalized tonic-clonic seizures. Epilepsia 2015; 56(9): 1408-14

[78] Kadel J, Bauer S, Hermsen AM et al. Use of Emergency Medication in Adult Patients with Epilepsy: A Multicentre Cohort Study from Germany. CNS Drugs 2018; 32(8): 771-81

[79] Strzelczyk A, Reese JP, Dodel R et al. Cost of epilepsy: a systematic review. PharmacoEconomics 2008; 26(6): 463-76

[80] Lagae L, Schoonjans AS, Gammaitoni AR et al. A pilot, open-label study of the effectiveness and tolerability of lowdose ZX008 (fenfluramine $\mathrm{HCl}$ ) in Lennox-Gastaut syndrome. Epilepsia 2018; 59(10): 1881-8

Bibliografie

DOI https://doi.org/10.1055/a-0987-8517

Nervenheilkunde 2019; 38: 887-899

(c) Georg Thieme Verlag KG Stuttgart · New York ISSN 0722-1541 


\section{Punkte sammeln auf CMF.thieme.de}

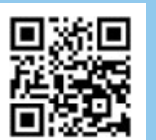

Diese Fortbildungseinheit ist bis zu 12 Monate online für die Teilnahme verfügbar.

Den genauen Einsendeschluss finden Sie beim Modul auf https://cme.thieme.de/CXDNDGQ.

Sollten Sie Fragen zur Online-Teilnahme haben, finden Sie unter https://cme.thieme.de/hilfe

eine ausführliche Anleitung. Wir wünschen viel Erfolg beim Beantworten

der Fragen!

Unter https://eref.thieme.de/CXDNDGQ oder über den QR-Code kommen Sie

direkt zum Artikel.

VNR 2760512019156640375

\section{Frage 1}

Welche Aussage zur Epilepsie ist falsch?

A Die Diagnose einer Epilepsie ergibt sich durch mindestens 3 unprovozierte epileptische Anfälle.

B In Deutschland sind etwa 0,6 bis $1 \%$ der Bevölkerung von Epilepsie betroffen.

C Das Vorliegen einer Narbe nach Schlaganfall oder eines Hirntumors sind Risikofaktoren für das Auftreten einer Epilepsie.

D Etwa 60 bis $80 \%$ der Menschen mit Epilepsie werden durch eine medikamentöse Therapie anfallsfrei.

E Bei pharmakoresistenter Epilepsie sollte eine epilepsiechirurgische Therapieoption geprüft werden.

\section{Frage 2}

Welche Aussage zur Kontraindikation für Antianfallsmedikamente ist falsch?

A Valproat sollte man bei Thrombopenie, Leberschädigung oder Pankreatitis nicht geben.

B Phenytoin kann schwere Herzrhythmusstörungen verursachen und sollte bei kardialer Instabilität nicht gegeben werden.

C Eine zusätzliche Therapie mit Meropenem kann bei Valproat zu einer deutlichen Erhöhung der Serumkonzentration und damit zu einer Toxizität führen.

D Levetiracetam ist für kardial vorerkrankte und instabile Patienten gut geeignet.

E Valproat kann unter einer Therapie mit Vitamin-K-Antagonisten zu einer Blutungsneigung führen.

\section{Frage 3}

Welches der folgenden Antianfallsmedikamente hat ein klinisch relevantes Interaktionspotenzial?
A Lacosamid
B Levetiracetam
C Pregabalin
D Carbamazepin
E Gabapentin

\section{Frage 4}

Welches Medikament kommt während einer Schwangerschaft am wenigsten in Betracht?
A Valproat
B Levetiracetam
C Oxcarbazepin
D Carbamazepin
E Lamotrigin

\section{Frage 5}

Welches Medikament gehört nicht zu den Natriumkanalblockern?
A Lacosamid
B Brivaracetam
C Oxcarbazepin
D Carbamazepin
E Lamotrigin

\section{Frage 6}

Welches Medikament ist nicht zur initialen Monotherapie fokaler Epilepsien zugelassen?
A Lacosamid
B Levetiracetam
C Oxcarbazepin
D Perampanel
E Lamotrigin

\section{Frage 7}

Welches Medikament ist nicht zur Therapie idiopathisch generalisierter Epilepsien zugelassen?
A Perampanel
B Levetiracetam
C Valproat
D Zonisamid
E Topiramat 


\section{Punkte sammeln auf CME.thieme.de}

Fortsetzung $\ldots$

\section{Frage 8}

Welche Aussage zum Status epilepticus ist falsch?

A Epileptische Anfälle dauern im Durchschnitt etwa 2 Minuten.

B Bei generalisiert tonisch-klonischen Anfällen ist ab einer Dauer von 5 Minuten von einem Status epilepticus auszugehen.

C Die Dringlichkeit der Therapie ist bei generalisiert konvulsiven Status epilepticus am höchsten.

D Der Status epilepticus tritt ausschließlich durch eine gestörte Erregungsleitung im Kleinhirn auf.

E Der Status epilepticus ist mit einer erhöhten Mortalität assoziiert.

\section{Frage 9}

Welche Substanz setzt man zur initialen Behandlung des Status epilepticus ein?
A Brivaracetam
B Lacosamid
C Benzodiazepine (z. B. Lorazepam)
D Thiopental
E Propofol

\section{Frage 10}

Welches Benzodiazepin ist zur Akuttherapie eines Status epilepticus ungeeignet?
A Midazolam
B Tetrazepam
C Lorazepam
D Diazepam
E Clonazepam 